\title{
Testes de rastreio de defeito cognitivo e demência: uma perspectiva prática
}

Manuela Guerreiro*

\section{RESUMO}

O diagnóstico precoce das demências degenerativas, particularmente da doença de Alzheimer (DA), é uma questão de grande interesse para investigadores e clínicos, sendo o conhecimento do perfil cognitivo na fase pré-clínica fundamental para o diagnóstico, para o prognóstico e para a intervenção farmacológica e não-farmacológica. Os médicos dos cuidados primários são os mais indicados para detectar precocemente o declínio cognitivo, embora alguns estudos revelem que a demência é subdiagnosticada nos cuidados primários de saúde. Isto deve-se à limitação de tempo que os médicos dispõem para as consultas e à falta de hábito na utilização de testes de rastreio cognitivo. Os testes de rastreio cognitivo devem ser rápidos de aplicar, fáceis de pontuar e bem aceites pelos doentes. Neste sentido, iremos referir alguns testes de aplicação rápida, mencionando algumas das suas características e alguns estudos onde foram utilizados. A aplicação de um teste breve poderá permitir a triagem de casos para posterior exame neuropsicológico detalhado, pois a detecção precoce da demência ainda em fases pré-clínicas e de Defeito Cognitivo Ligeiro ( $\mathrm{DCL}$ ) nos idosos é uma prioridade na saúde pública, devido à elevada incidência de demências.

Palavras-chave: Testes Cognitivos Breves; Rastreio; Defeito Cognitivo Ligeiro; Demência ligeira.

\section{INTRODUÇÃO}

A pesar do aumento do conhecimento sobre a fisiopatologia das demências, particularmente da DA, o diagnóstico continua a depender em grande parte da opinião clínica e dos resultados da avaliação neuropsicológica. No entanto, a fase inicial de demência não é frequentemente identificada e o diagnóstico, muitas vezes, só é feito dois ou três anos após os primeiros sintomas. ${ }^{1}$ Quando se faz a triagem em sujeitos que não são sintomáticos, encontra-se uma taxa elevada de casos falsos positivos, pelo que muitos investigadores recomendam que o rastreio seja feito em sujeitos sintomáticos. No entanto, o diagnóstico precoce é extremamente importante e o rastreio deverá ser realizado sempre que existam queixas de dificuldades mnésicas. ${ }^{2-5}$

As alterações cognitivas são heterogéneas nas fases iniciais das demências degenerativas, mas a memória episódica e/ou as funções executivas estão geralmen-

* Investigadora Auxiliar - Laboratório de Estudos de Linguagem - Faculdade de Medicina de Lisboa. te alteradas em fases muito precoces, nomeadamente, na fase de «Defeito Cognitivo Ligeiro» (DCL). ${ }^{6}$ Nesta fase o doente refere geralmente dificuldades de memória, as quais não são muitas vezes valorizadas pelos médicos porque o doente mantém o seu funcionamento quotidiano ou porque as queixas de dificuldades mnésicas são demasiado frequentes na população idosa e poderão estar associadas ao próprio envelhecimento normal ou a quadros de sintomatologia depressiva..$^{7-9} \mathrm{O}$ problema coloca-se em como objectivar e valorizar estas queixas, de uma forma simples e rápida, numa consulta de rotina. Vários testes breves têm sido desenvolvidos, todos eles apresentando limitações, mas que poderão ajudar o clínico a decidir se deve ou não pedir um exame neuropsicológico exaustivo, ou referenciar a uma consulta da especialidade. $O$ uso de testes breves de triagem usados na medicina geral e familiar podem constituir instrumentos de utilidade para a identificação de alterações cognitivas, contribuindo para a identificação precoce de pessoas com DCL ou demência e início de tratamento farmacológico e/ou não-farmacológico para atrasar a progressão, bem como para es- 
tabelecer medidas que reduzam o impacto social da doença.

Os médicos de clínica geral podem desempenhar um papel fundamental na detecção precoce de pessoas com sintomas sugestivos de demência, desde que disponham de instrumentos de aplicação rápida para o rastreio destes casos.

\section{AVALIAÇÃO DO «DEFEITO COGNITIVO LIGEIRO» - ASPECTOS GERAIS}

O conceito de «Defeito Cognitivo Ligeiro» (DCL) implica a existência de queixas de memória e a objectivação desse défice em testes de memória. As restantes funções cognitivas estão relativamente preservadas, assim como o desempenho das actividades instrumentais da vida diária. Porém, cerca de 10 a 15\% dos sujeitos com DCL desenvolvem DA por ano. ${ }^{10}$

Muitos trabalhos tentam seleccionar os melhores instrumentos para o diagnóstico de DCL e os que melhor prevêem a evolução de DCL para demência. Desempenhos baixos em tarefas de memória episódica e em testes de funções executivas podem ter valor preditivo na evolução para DA. ${ }^{11,12}$ Estudos longitudinais mostram, pelo seguimento dos sujeitos com DCL, que a pontuação baixa em testes de recordação de palavras, de reconhecimento de faces e de fluência verbal, poderão ser preditores fiáveis do início de demência, desde que a pontuação no teste breve de avaliação do estado mental (Mini Mental State Examination - MMSE) ${ }^{13}$ seja tida em conta. ${ }^{14}$. Diniz et al (2008) ${ }^{15}$ usaram em simultâneo o MMSE e o "Cambridge Cognitive Examination ${ }^{16}$ num grupo de doentes com DA ligeira, DCL e controlos, tendo concluído que estes testes discriminavam adequadamente casos de DA ligeira de controlos, mas não revelavam precisão para discriminar entre controlos e doentes com DCL. Esta discriminação só era conseguida com uma avaliação neuropsicológica exaustiva. Este trabalho sugere que a identificação de doentes com DCL requer uma avaliação mais detalhada do que só a avaliação da memória e que é necessário desenvolver novas estratégias para o rastreio de défices cognitivos ligeiros na prática clínica.

Outro aspecto importante a ter em atenção é, por um lado, o facto de o défice cognitivo no idoso poder estar associado à depressão e, por outro lado, ser frequente a presença de sintomas depressivos nos sujeitos com
DCL ou com DA inicial, levantando-se assim a questão do diagnóstico diferencial entre DCL ou demência inicial versus depressão. ${ }^{8,9}$ No entanto, uma análise de desempenhos qualitativos e de memória episódica evidenciou diferenças que podem ser úteis no diagnóstico diferencial entre DA e depressão. As alterações na memória em doentes com depressão estão relacionadas com mecanismos de recuperação da informação e não de codificação, dependentes das funções executivas, cuja disfunção se associa à gravidade da depressão. ${ }^{17}$

\section{AVALIAÇÃO DA DEMÊNCIA - ASPECTOS GERAIS}

Para o diagnóstico de demência ser estabelecido é necessário que exista já diminuição no desempenho funcional, para além de défices em várias capacidades cognitivas, embora na maioria dos casos com demência o declínio na capacidade funcional esteja fortemente relacionado com o desempenho cognitivo, principalmente com as funções executivas. ${ }^{18}$

Para avaliar a capacidade de desempenhar actividades de vida diária, existem muitas escalas cujo preenchimento se baseia em auto-relatos do doente e/ou em informações fornecidas pela família ou pelos acompanhantes do doente. Entre estas escalas, referimos a escala de demência de Blessed ${ }^{19-21}$ e a escala de Actividades Instrumentais de Vida Diária (AIVD) de Lawton. ${ }^{19,22}$ Apesar de a informação fornecida pelas escalas ser fundamental para o diagnóstico, não nos podemos esquecer que poderá existir um viés no seu preenchimento e que as escalas poderão também ser pouco sensíveis a mudanças subtis que ocorrem no DCL e nas fases incipientes da DA e de outras demências..$^{23}$ No entanto, muitos autores recomendam o seu uso, e alguns trabalhos revelam a superioridade das escalas funcionais em relação aos testes cognitivos breves na detecção precoce de demência em sujeitos de baixa escolaridade.

\section{TESTES BREVES PARA AVALIAÇÃO DO ESTADO MENTAL}

\section{Exame Breve do Estado Mental (MMSE)}

Entre todos os testes breves para avaliação do estado mental, o Exame Breve do Estado Mental ${ }^{19}$, conhecido por MMS (Mini Mental State) ou MMSE (Mini Mental State Examination) ${ }^{13}$ é talvez o mais usado na literatura internacional. Embora este teste não deva ser apli- 
cado para fazer diagnósticos formais, ${ }^{24}$ pode ser utilizado como um primeiro passo para a detecção de declínio cognitivo. ${ }^{25-27}$ No entanto, os valores dos pontos de corte têm de ser adaptados à população, pois os valores originais dos testes (ponto de corte de 24, num máximo de 30 pontos) não se revelam adequados para uma boa precisão na discriminação de sujeitos com e sem défice cognitivo. ${ }^{19,28}$ Num trabalho recente de validação do MMS, ${ }^{29}$ foi encontrado efeito do género na população com escolaridade baixa, mas os autores não referem a relação com a idade, podendo o efeito de género ser secundário à relação escolaridade/idade (nos grupos mais idosos, as mulheres, geralmente, apresentam escolaridade mais baixa), como verificámos aquando da validação na população portuguesa. ${ }^{28}$

O MMSE revela boa concordância entre a aplicação por clínicos gerais e por neuropsicólogos e alta fiabilidade teste-reteste. ${ }^{30,31}$ Também o declínio no MMSE entre duas observações pode ser um indicador de evolução para demência. Numa população com idade superior a 74 anos e sem demência, foi encontrado declínio de um ponto no MMSE entre duas observações com um ano e meio de diferença, e essa modificação revelou associação significativa com a evolução dos casos para demência. ${ }^{32}$ Num estudo de meta-análise sobre a precisão de diagnóstico do MMSE na detecção de demência e DCL, foram encontrados valores de sensibilidade e de especificidade diferentes, dependendo de os estudos serem realizados em clínicas de memória, em consultas hospitalares, na comunidade não-clínica ou nos cuidados primários de saúde. Esta revisão incluiu 34 estudos, dos quais apenas cinco comparavam DCL com sujeitos saudáveis e três comparavam DCL com DA, concluindo que o MMSE apresenta uma precisão diagnóstica baixa, embora com valores melhores para excluir demência em estudos na comunidade e cuidados primários. Noutras situações, é sugerido que o MMSE seja combinado com outros métodos ou mesmo substituído por outros instrumentos. ${ }^{33}$

O MMSE é um instrumento de aplicação rápida (menos de 10 minutos) para a avaliação do desempenho cognitivo. Examina a orientação no tempo e no espaço, memória, atenção, linguagem oral (nomeação e compreensão) e escrita. A pontuação total é de 30 pontos. De acordo com a padronização na População Portuguesa, foram encontrados valores de ponto de corte diferentes em função da escolaridade, de modo a permitir diferenciar entre pessoas com e sem défice cognitivo. O MMSE pode apresentar um efeito de tecto no DCL e um efeito de chão em demências graves. O efeito de tecto (falsos negativos) poderá ser encontrado em sujeitos com um elevado QI pré-mórbido e em pessoas com demência não-DA (por exemplo, demência de corpos de Lewy ou demência frontotemporal), as quais podem obter pontuações superiores às dos pontos de corte. Para além dos efeitos da escolaridade, também poderão ser encontrados efeitos do tipo de profissão. ${ }^{34}$ Estes problemas colocam-se, de resto, em todos os testes neuropsicológicos. As medidas globais, de tipo escalas, estão menos sujeitas a influências culturais ou aos efeitos da prática, mas são mais subjectivas. Principalmente em avaliações de seguimento, as escalas globais apresentam superioridade em relação ao MMSE e em ensaios clínicos estas medidas têm sido mais úteis na avaliação da eficácia. ${ }^{35}$

As medidas globais são baseadas na entrevista clínica e incluem as escalas de gravidade global e as escalas de mudanças globais. Nas escalas de gravidade global, estão incluídas, por exemplo, a «Clinical Dementia Rating» (CDR $)^{19,36,37}$ e a escala de Deterioração Global (GDS). ${ }^{19,38,39}$ As escalas de gravidade global são relativamente livres de alguns vieses sócio-culturais e podem ser usadas para demonstrar a eficácia terapêutica em termos da progressão geral do processo demencial. ${ }^{39}$

Num estudo que analisou se a medida de actividades instrumentais de vida diária podia detectar diferenças entre doentes com DCL e idosos normais foi encontrado que os sujeitos com DCL apresentavam pontuações significativamente inferiores numa escala de Actividades Instrumentais de Vida Diária (AIVD) ${ }^{40}$, com declínio de um ponto num ano, em $50 \%$ dos doentes, em comparação com um declínio semelhante só em $29 \%$ dos sujeitos controlos. ${ }^{41}$

\section{Montreal Cognitive Assessment (MoCA)}

Um outro instrumento breve, e que se encontra validado para a população portuguesa, é o «Montreal Cognitive Assessment» (MoCA). ${ }^{42,43} \mathrm{O}$ MoCA é um teste que demora cerca de 10 minutos, com 30 pontos de pontuação máxima. Foi concebido como teste de rastreio cognitivo para ajudar os profissionais de saúde na detecção do DCL, principalmente em doentes com pontua- 
ção entre 24 e 30 pontos no MMSE. O ponto de corte sugerido no MoCA é de 26 pontos. Este teste tem mais ênfase nas tarefas de funcionamento executivo e de atenção do que o MMSE, o que pode torná-lo mais sensível na detecção de demências não-DA.

Smith et al. (2007) ${ }^{44}$ aplicaram o MoCA e o Exame Breve do Estado Mental (MMSE) para detecção da sensibilidade e especificidade do teste em indivíduos que preenchiam os critérios de diagnóstico de demência e de DCL, com acompanhamento de seis meses. Verificaram que, para um ponto de corte de 26, o MMSE apresentava sensibilidade de $17 \%$ para detectar indivíduos com a DCL, enquanto que o MoCA detectou $83 \%$. O MMSE apresentou sensibilidade de $25 \%$ para detectar indivíduos com demência, enquanto a MoCA detectou $94 \%$, sendo a especificidade para o MMSE de $100 \%$ e para o MoCA foi de $50 \%$. Os autores concluem que o teste é um instrumento útil para rastreio rápido de demência ligeira ou mesmo de DCL. No entanto, não foram encontradas diferenças significativas entre o MoCA e o MMSE, na avaliação de modificações cognitivas ao longo do período de 6 meses.

\section{Desenho do Relógio - Clock Drawing Test (CDT).}

O Exame Breve de Estado Mental (MMSE) muitas vezes não consegue identificar a disfunção executiva, mesmo quando esta é grave. Os exames neuropsicológicos e testes de cabeceira mais exaustivos podem avaliar a função executiva com mais especificidade, mas requerem demasiado tempo. O teste do relógio é usado nesta perspectiva, apesar de apresentar limitações. ${ }^{45,46} \mathrm{~A}$ disfunção executiva pode ter um impacto significativo na capacidade de decisão, mas é particularmente difícil de diagnosticar em sujeitos com um MMSE normal.

O desenho do relógio (Clock Drawing Test-CDT) é amplamente utilizado para triagem de demência, mas é questionável se é adequado para o rastreio de DCL. Ehreke et al. (2009) ${ }^{47}$ estudaram com exame neuropsicológico detalhado cerca de 3.000 doentes de cuidados primários de saúde com idade superior a 75 anos, considerando dois grupos: os que tinham DCL e os que não tinham quaisquer alterações cognitivas. As pontuações do CDT de ambos os grupos foram comparadas e revelaram diferenças significativas, tendo os doentes com DCL obtido resultados piores do que os normais. Contudo, a sensibilidade e especificidade, bem como ou- tras características psicométricas, não se revelaram adequadas segundo estes autores para que o CDT possa ser considerado um instrumento de eleição no contexto da avaliação breve do estado mental. A utilização do CDT em conjunto com o MMSE para detectar casos de DCL e para discriminar casos com demência ligeira de não-dementes também não revela precisão diagnóstica. Contudo, o CDT é frequentemente utilizado na prática clínica para o rastreio de distúrbios cognitivos. Numa revisão sistemática da literatura Pinto e Peters $(2009)^{48}$ encontraram boa fiabilidade inter-observadores, em teste-reteste e boa correlação com outros testes de rastreio, mas o teste revela grande influência da idade e escolaridade. Também demonstra baixa sensibilidade e especificidade, muito variável em doentes com demência ligeira ou questionável, embora tenha características de um bom método de rastreio em demências moderadas ou graves. Numa revisão da literatura foram encontrados cinco estudos que utilizaram o CDT como preditor. Apesar dos dados serem díspares, os autores concluem que o teste pode ser um instrumento útil para identificar declínio cognitivo antes de outros testes mais tradicionais. ${ }^{49}$

Alguns métodos de aplicação do teste CDT implicam a instrução de desenhar o relógio marcando uma determinada hora e outros apenas solicitam o desenho do relógio. O método que solicita a indicação da hora, parece apresentar maior sensibilidade, maior valor preditivo e uma percentagem inferior de mal-classificados do que o método que solicita apenas o desenho do relógio, sem pedir a indicação de hora. As análises qualitativas dos erros na indicação da hora reflectem alterações no pensamento abstracto e conceptual e ocorrem, quase exclusivamente, em casos de demência. ${ }^{50}$

Em sujeitos com idade superior a 64 anos, com demência e com escolaridade igual ou inferior a 4 anos, o teste é pouco fidedigno, não sendo adequado para a triagem de demência neste grupo. ${ }^{51}$ Juby et al. (2002) ${ }^{46}$ investigaram o valor do CDT em complemento com o MMSE na identificação da possível disfunção executiva em doentes em situação de consulta médica e verificaram que, de $97 \%$ dos doentes com alterações no MMSE, 83\% apresentam também défice na pontuação do desenho do relógio. Dos doentes com MMSE normal, 50\% tinham alterações no CDT. Os autores consideram que a combinação do CDT com o MMSE poderá ajudar na detecção 
de sujeitos com défice cognitivo na prática clínica. No entanto, tal como o MMSE, o desempenho no CDT pode estar muito relacionado com a escolaridade. O estudo de Juby et al. $(2002)^{46}$ mostra que um MMSE com pontuação normal não exclui a disfunção cognitiva e que a adição do CDT pode melhorar a avaliação dos domínios da cognição não abrangidos pelo MMSE sozinho.

A validação do desenho do relógio para a população portuguesa foi feita na versão mais simples: «Desenhe um relógio redondo com números e ponteiros», sendo a pontuação de zero a três pontos, em que o zero corresponde a um desenho quase irreconhecível e a pontuação de três corresponde ao desenho correcto (mostrador redondo, números na sequência correcta e simétricos: o seis em relação ao doze e o três em relação ao nove, e a presença de dois ponteiros). ${ }^{20}$

\section{Testes de Fluência Verbal}

Os testes de fluência verbal também são utilizados como testes de rastreio. A sua aplicação é de um minuto e a pontuação corresponde ao número de itens evocados nesse minuto.

Sebaldt et al. (2009) ${ }^{52}$ compararam os resultados de um teste de fluência verbal para nomes de animais com os resultados obtidos no MoCA, em sujeitos com idade superior a 64 anos e com factores de risco vascular sem defeito cognitivo aparente, da consulta de cuidados primários. O teste de Fluência Verbal (animais) (FVA) e o MoCA detectaram defeito cognitivo em $52 \%$ e $56 \%$, respectivamente, da população estudada. O teste FVA demonstrou sensibilidade, especificidade e precisão, pelo que poderá ser útil na detecção de defeito cognitivo ou de demência. O tempo curto de aplicação do teste de FVA é a favor da sua inclusão na prática clínica.

O teste de fluência verbal para produtos alimentares («diga-me que produtos alimentares pode comprar na mercearia ou no supermercado») encontra-se aferido para a população portuguesa, para sujeitos com idade superior a 50 anos, apresentando valores diferentes em função da escolaridade (valores mínimos normais: analfabetos $=8$; até 4 anos de escolaridade $=12$; acima de 4 anos de escolaridade $=14) .{ }^{20}$

\section{Questionnaire on Cognitive Decline in the Elderly (IQCODE)}

A triagem da demência ligeira ou de DCL em idosos de muito baixa escolaridade é difícil. Nestes casos, a informação dos acompanhantes e dos familiares usando versões longa ( 26 itens) ou breve (16 itens) do «Questionário de Declínio Cognitivo em Idosos» pode ser de grande utilidade. ${ }^{53,54}$ Perroco et al. (2009) ${ }^{55}$ investigaram as duas versões e uma outra versão Brasileira (15 itens), em comparação com o MMSE, no rastreio de demência numa população idosa com escolaridade baixa. A escolaridade apresentou correlação com o MMSE, mas não com as versões dos IQCODE. Neste estudo, o MMSE classificou correctamente $85,7 \%$ dos indivíduos, enquanto as três versões IQCODE classificaram correctamente $91,2 \%$ dos sujeitos. Qualquer das versões do IQCODE pode ser instrumento de triagem útil para doentes com demência ligeira ou moderada. O IQCODE apresenta a grande vantagem de não ser influenciado pela escolaridade e parece ser um instrumento útil para populações com baixo nível de escolaridade.

O IQCODE é rápido e fácil de usar, revelando boa sensibilidade $(0,86)$ para o diagnóstico de demência, mas baixa especificidade $(0,39)$ e baixo valor preditivo positivo. Contudo, a combinação do IQCODE com o MMSE melhora consideravelmente a precisão, a especificidade e o valor preditivo positivo, sendo esta combinação de utilidade diagnóstica superior à utilização só do IQCODE. ${ }^{56}$

\section{O Teste dos Três Objectos}

\section{(Three-Objects-Three-Places Test) (303P)}

Nos exames neuropsicológicos, a memória episódica é tipicamente avaliada com testes de evocação e reconhecimento de algumas informações em contexto experimental (por exemplo, o teste de recordação livre, recordação da Figura de Rey ou o teste de memória verbal de Rey). ${ }^{57-59}$ No entanto, os testes clássicos têm sido criticados por terem pouca validade ecológica. ${ }^{60,61}$ Prestia et al. (2006) ${ }^{62}$ desenvolveram e validaram um instrumento ecológico de triagem, o chamado «Teste Neuropsicológico dos 3-Objectos-3-Locais» (3O3P), que envolve principalmente a memória episódica e que foi desenvolvido como um teste ecológico curto de memória episódica para o rastreio da doença de Alzheimer (AD). Este teste foi validado em doentes com DA e com nãoDA, doentes com DCL e indivíduos normais.

O teste 303P é simples e rápido. Primeiro, o examinador mostra ao doente três objectos (um lápis, uma 
chave e uma moeda) e pede para os nomear (a fim de verificar se há défice de produção da linguagem ou no reconhecimento de objectos que, se presente, torna o teste impossível de ser executado). Em seguida, o clínico esconde os objectos em três lugares diferentes (o lápis atrás de um telefone a cerca de $1 \mathrm{~m}$ de distância, a chave de um armário a $2 \mathrm{~m}$ de distância, e a moeda dentro de um livro a 1,5 m de distância do doente), de modo a que os objectos não estejam visíveis, mas certificando-se que o doente viu onde os objectos foram escondidos. Depois de 5 minutos (poderá pedir-se o desenho do relógio ou aplicar-se outro teste breve), o médico pede ao paciente para: 1- se lembrar do nome dos objectos que viu, 2- dizer os locais onde foram escondidos e 3- dizer as correspondências correctas entre objectos e locais. A pontuação é de 1 ponto para cada resposta correcta (intervalo de $0-9$ pontos). $\mathrm{O}$ teste revelou uma especificidade entre $87 \%$ e $91 \%$ e uma sensibilidade entre $92 \%$ e $100 \%$ na identificação correcta de DA, em idades compreendidas entre os 50 a 65 anos e 66 a 80 anos. O teste 3O3P apresenta alta homogeneidade e fiabilidade e boa consistência interna. Além disso, mostra sensibilidade muito alta na detecção de doentes com DA, embora seja menos preciso na discriminação entre doentes com demência DA e doentes com demência não-DA. O teste 3O3P é simples e interactivo e pode ser útil com pessoas idosas, que muitas vezes se sentem desconfortáveis com tarefas relativamente difíceis como as dos testes clássicos. $\mathrm{O}$ teste ecológico 303P é rápido, preenche os critérios de um instrumento para rastreio cognitivo e poderá ser usado por diferentes profissionais, nomeadamente, nas consultas de Cuidados de Saúde Primários.

\section{CONCLUSÃO}

O MMSE ${ }^{13,19}$ é o teste mais utilizado na literatura para rastreio de demência e é usado como teste de referência para a validação de muitos outros testes breves. No entanto, na prática clínica dos Cuidados de Saúde Primários, poderá ainda ser considerado demasiado longo. Por outro lado, para a detecção de DCL, o MMSE não tem mostrado poder discriminativo satisfatório entre sujeitos normais e sujeitos com DCL, o que é melhor conseguido com o teste MoCA. ${ }^{42,43}$ Num trabalho de revisão, que analisou 15 testes breves de rastreio, valores adequados de sensibilidade e de especificidade foram en- contrados apenas em 4 testes, entre eles o MoCA. ${ }^{63}$ Este trabalho mostra que a capacidade do MMSE para discriminar sujeitos com DCL de controlos idosos saudáveis não está estabelecida de forma consistente, variando com os diferentes valores usados como ponto de corte, e que a sensibilidade do MMSE para o DCL é geralmente baixa, embora com algumas excepções, mas a sua especificidade é alta. ${ }^{64}$ No entanto, embora isoladamente as sensibilidades do MMSE e do desenho do relógio sejam baixas para o diagnóstico de DCL, aumentam para $75 \%$ quando os testes são usados em conjunto. ${ }^{33}$

Dentro da variedade de testes mais breves, consideramos que será importante o médico seleccionar o teste, ou os testes, que sente como mais confortáveis para aplicar. Uma possibilidade será usar o teste dos três objectos, isolado ou em combinação com a fluência verbal para produtos alimentares, o que terá um tempo de aplicação inferior a 5 minutos. O teste do relógio é bastante sensível ao défice cognitivo, mas só deve ser aplicado a sujeitos com escolaridade superior a 4 anos. Mesmo quando há a possibilidade de aplicar o MMSE ou o MoCA, que estão validados na população portuguesa, os resultados serão mais precisos se for adicionado um outro teste rápido, como, por exemplo, a fluência verbal. O uso de uma escala funcional poderá fornecer indicadores importantes para o diagnóstico precoce de demência, particularmente se for considerada a informação fornecida por um acompanhante que seja próximo do doente (cônjuge, filhos ou alguém que contacte no dia-a-dia com o doente). A opção de triagem pode também passar pelo uso de uma escala funcional em simultâneo com um teste muito breve pois, particularmente em sujeitos de baixa escolaridade, o valor das escalas funcionais pode superar a precisão de um teste breve no diagnóstico precoce da demência.

Pode concluir-se que não há consenso quanto ao melhor teste cognitivo para usar quando há suspeita de DCL ou demência. No entanto, a prática no uso de um determinado instrumento conduz a um melhor conhecimento desse instrumento e a uma melhor capacidade na interpretação dos seus resultados, pelo que é fundamental que os Médicos de Família optem por introduzir nas suas consultas um instrumento breve de triagem cognitiva, pois estão em situação privilegiada para reconhecer precocemente casos de défice cognitivo. 


\section{REFERÊNCIAS BIBLIOGRÁFICAS}

1. Aguero-Torres H, Fratiglioni L, Winblad B. Natural history of Alzheimer's disease and other dementias: review of the literature in the light of the findings from the Kungsholmen Project. Int J Geriatr Psychiatry 1998;13(11):755-66.

2. Raschetti R, Maggini M, Sorrentino GC, Martini N, Caffari B, Vanacore N. A cohort study of effectiveness of acetylcholinesterase inhibitors in Alzheimer's disease. Eur J Clin Pharmacol 2005;61(5-6):361-8.

3. Lowenstein DA, Acevedo A, Czaja SJ, Duara R. Cognitive rehabilitation of mildly impaired Alzheimer disease patients on cholinesterase inhibitors. Am J Geriatr Psychiatry 2004;12:395-402.

4. Olazaran J, Muniz R, Reisberg B, Peña-Casanova J, del Ser T, Cruz-Jentoft AJ, Serrano P, Navarro E, García de la Rocha ML, FrankA, Galiano M, Fernández- Bullido Y, Serra JA, González-Salvador MT, Sevilla C. Benefits of cognitive-motor intervention in $\mathrm{MCl}$ and mild to moderate Alzheimer disease. Neurology 2004;63(12):2348-53.

5. Spector A, Thorgrimsen L, Woods B, Royan L, Davies S, Butterworth M, Orrell M. Efficacy of an evidence-based cognitive stimulation therapy programme for people with dementia: randomised controlled trial. Br J Psychiatry 2003;183:248-54.

6. Traykov L, Raoux N, Latour F, Gallo L, Hanon O, Baudic S, Bayle C, Wenisch E, Remy P, Rigaud AS. Executive functions deficit in mild cognitive impairment. Cogn Behav Neurol. 2007 Dec;20(4):219-24.

7. Deary IJ, Corley J, Gow AJ, Harris SE, Houlihan LM, Marioni RE, Penke L, Rafnsson SB, Starr JM. Age-associated cognitive decline. Med Bull. 2009;92:135-52.

8. Dillon C,Allegri RF, Serrano CM, Iturry M, Salgado P, Glaser FB, Taragano FE. Lateversus early-onset geriatric depression in a memory research center. Neuropsychiatr Dis Treat. 2009;5:517-26.

9. Herrmann LL, Goodwin GM, Ebmeier KP. The cognitive neuropsychology of depression in the elderly. Psychol Med. 2007 Dec;37(12):1693-702.

10. Petersen RC, Doody R, Kurz A, Mohs RC, Morris JC, Rabins PV, Ritchie K, Rossor $M$, Thal L, Winblad B. Current concepts in mild cognitive impairment. Arch Neurol 2001 Dec;58(12):1985-92.

11. Arnaiz E,Almkvist O. Neuropsychological features of mild cognitive impairment and preclinical Alzheimer's disease. Acta Neurol Scand 2003;179: 34-41.

12. Marra C, Silveri M, Gainotti G. Predictors of cognitive decline in the early stage of probable Alzheimer's disease. Dement Geriatr Cogn Disord 2000;11:212-8.

13. Folstein M, Folstein M, McHugh P. 'Mini-Mental State' - A practical method for grading the cognitive state of patients for the clinician. J Psychiatr Res 1975; 12:189-98.

14. Chen P, Ratcliff G, Belle SH, Cauley JA, DeKosky ST, Ganguli M. Cognitive tests that best discriminate between presymptomatic $A D$ and those who remain nondemented. Neurology 2000 Dec 26;55(12):1847-53.

15. Diniz BS, Nunes PV, Yassuda MS, Pereira FS, Flaks MK, Viola LF, Radanovic M, Abreu ID, Borelli DT, Gattaz WF, Forlenza OV. Mild cognitive impairment: cognitive screening or neuropsychological assessment? Rev Bras Psiquiatr 2008 Dec;30(4):316-21.

16. Roth M, Tym E, Mountjoy CQ, Huppert FA, Hendrie H,Verma S, Goddard R. CAMDEX.A standardized instrument for the diagnosis of mental disorders in the elderly with special reference to early detection of dementia. Br J Psychiatry 1986; 149:698-09.

17. Baudic S, Tzortzis C, Barba GD, Traykov L. Executive deficits in elderly patients with major unipolar depression.J Geriatr Psychiatry Neurol 2004 Dec;17(4):195201.

18. Goverover Y. Categorization, deductive reasoning, and selfawareness: association with everyday competence in persons with acute brain injury. J Clin Exp Neuropsychol 2004;26(6):737-49.

19. Grupo de Estudos de Envelhecimento Cerebral e Demência. Escalas e testes na demência. Colaboração Novartis. $2^{\mathrm{a}}$ Ediçãa; 2008.

20. Garcia C. A Doença de Alzheimer: problemas do diagnóstico clínico - Tese de Doutoramento. Lisboa: Faculdade de Medicina de Lisboa; 1984.

21. Blessed G, Tomlinson BE, Roth M... Association between quantitative measures of dementing and senile change in the cerebral gray matter of elderly subjects. Br J Psychiatry 1968;114:797-11.

22. Lawton MP, Brody EM. Assessment of older people: self-maintaining and instrumental activities of daily living. Gerontologist. 1969; 9(3):179-86.

23. Lowenstein $A D$. A new scale for the assessment of functional status in $A D$ and related disorders. J Gerontol 1989;44(4):114-21.

24. O'Connor DW, Pollitt PA, Treasure FP, Brook CP, Reiss BB. The influence of education, social class and sex on Mini-Mental State scores. Psychol Med 1989;19(3):771-6.

25. Fabrigoule C, Lechevallier N, Crasborn L, Dartigues JF, Orgogozo JM. Inter-rater reliability of scales and tests used to measure mild cognitive impairment by general practitioners and psychologists. Curr Med Res Opin 2003;19(7):603-8.

26. Tierney MC, Szalai JP, Dunn E, Geslani D, McDowell I. Prediction of probable Alzheimer disease in patients with symptoms suggestive of memory impairment. Arch Fam Med 2000;9(6):527-32.

27. Tangalos EG, Smith GE, lvnik RJ, Petersen RC, Kokmen E, Kurland LT, et al. The Mini-Mental State Examination in general medical practice: clinical utility and acceptance. Mayo Clin Proc 1996;71(9):829-37.

28. Guerreiro M, Silva AP, Botelho MA, Leitão O, Garcia C. Adaptação à população portuguesa da tradução do "Mini Mental State Examination" (MMSE). Rev Port Neurol 1994;9-10.

29. Ibrahim NM, Shohaimi S, Chong HT, Rahman AH, Razali R, Esther E, Basri HB. Validation study of the Mini-Mental State Examination in a Malay-speaking elderly population in Malaysia. Dement Geriatr Cogn Disord 2009;27(3):247-53.

30. Pezzotti P, Scalmana S, Mastromattei A, Di Lallo Domenico. The accuracy of the MMSE in detecting cognitive impairment when administered by general practioners: A prospective observational study. BMC Family Practice 2008;9(29).

31. Magni E, Binetti G, Bianchetti A, Rozzini R, Trabucchi M. Mini-mental State Examination: a normative study in Italian elderly population. Eur J Neurol 1996;3:1-5.

32. Hensel A, LuckT, Luppa M, Glaesmer H,Angermeyer MC, Riedel-Heller SG. Does a reliable decline in Mini Mental State Examination total score predict dementia? Diagnostic accuracy of two reliable change indices. Dement Geriatr Cogn Disord. 2009;27(1):50-8.

33. Mitchell AJ. A meta-analysis of the accuracy of the mini-mental state examination in the detection of dementia and mild cognitive impairment.J Psych Res 2009;43:411-31.

34. Frisoni GB, Rozzini R, Bianchetti A, Trabucchi M. Principal lifetime occupation and MMSE score in elderly persons. J Gerontol 1993 Nov;48(6):S310-4.

35. Reisberg B. Global measures: utility in defining and measuring treatment response in dementia. Int Psychogeriatr 2007 Jun;19(3):421-56.

36. Hughes CP, Berg L, Danziger WL, Coben LA, Martin RL. A new clinical scale for the staging of dementia. Br J Psychiatry 1982 Jun;140:566-72.

37. Morris J. The CDR: current version and scoring rules. Neurology 1993;43: 241213.

38. Reisberg B, Ferris SH, Leon MJ, Crook T. The Global Deterioration Scale (GDS) for assessment of primary degenerative dementia. Am J Psych 1982;139: 1136-9.

39. Reisberg B, Ferris S, Schulman E, Steinberg G, Buttinger C, Sinaiko E, et al. Longitudinal course of normal aging and progressive dementia of Alzheimer's type: a prospective study of 106 subjects over a 3.6 year mean interval. Prog Neuropsychopharmacol Biol Psychiatry 1986;10(3-5):571-8.

40. Cullum CM, Saine K, Chan LD, Martin-Cook K, Gray KF, Weiner MF. Performancebased instrument to assess functional capacity in dementia: The Texas Functional Living Scale. Neuropsychiatr Neuropsychol Behav Neurol 2001;14:103-108.

41. Binegar DL, Hynan LS, Lacritz LH, Weiner MF, Cullum CM. Can a direct IADL measure detect deficits in persons with MCI? Curr Alzheimer Res 2009 Feb;6(1):4851.

42. Nasreddine ZS, Phillips NA, Bédirian V, Charbonneau S, Whitehead V, Collin I, et al. The Montreal Cognitive Assessment, MoCA: a brief screening tool for mild cognitive impairment. J Am Geriatr Soc 2005;53(4):695-9. 
43. Duro D, Simões MR, Ponciano E, Santana I. Validation studies of the Portuguese experimental version of the Montreal Cognitive Assessment (MoCA): confirmatory factor analysis. J Neurol [publicação online] 2009 Nov 25 [Acedido a: 14/12/2009].

44. Smith T, Gildeh N, Holmes C. The Montreal Cognitive Assessment: validity and utility in a memory clinic setting. Can J Psychiatry 2007 May;52(5):329-32.

45. Eddy JR, Sriram S. Clock-drawing and telling time as diagnostic aids. Neurology 1977 Jun;27(6):595.

46. Juby A, Tench S, BakerV.The value of clock drawing in identifying executive cognitive dysfunction in people with a normal Mini-Mental State Examination score. CMAJ 2002;167(8):859-64.

47. Ehreke L, Luppa M, LuckT, Wiese B, Weyerer S, Eifflaender-Gorfer S, et al. Is the clock drawing test appropriate for screening for mild cognitive impairment? Results of the German study on Ageing, Cognition and Dementia in primary care patients (AgeCoDe). Dement Geriatr Cogn Disord 2009;28(4):365-72.

48. Pinto E, Peters R. Literature review of the clock drawing test as a tool for cognitive screening. Dement Geriatr Cogn Disord 2009;27(3):201-13.

49. Peters R, Pinto EM. Predictive value of the clock drawing test. A review of the literature. Dement Geriatr Cogn Disord 2008;26(4):351-5.

50. Berger G, Frölich L, Weber B, Pantel J. Diagnostic accuracy of the clock drawing test: the relevance of "time setting" in screening for dementia. J Geriatr Psychiatry Neurol 2008 Dec;21(4):250-60.

51. Lourenço RA, Ribeiro-Filho ST, Moreira Ide F, Paradela EM, Miranda AS. The clock drawing test: performance among elderly with low educational level. Rev Bras Psiquiatr 2008 Dec;30(4):309-15.

52. Sebaldt R, Dalziel W, Massoud F, Tanguay A, Ward R, Thabane L, et al. Detection of cognitive impairment and dementia using the animal fluency test: the DECIDE study. Can J Neurol Sci 2009 Sep;36(5):599-604

53. Jorm AF, Jacomb PA. The Informant Questionnaire on Cognitive Decline in the Elderly (IQCODE): socio-demographic correlates, reliability, validity and some norms. Psychol Med 1989 Nov;19(4):1015-22.

54. Jorm AF, Scott R, Cullen JS, MacKinnon AJ. Performance of the Informant Questionnaire on Cognitive Decline in the Elderly (IQCODE) as a screening test for dementia. Psychol Med 1991 Aug;21(3):785-90.

55. Perroco TR, Bustamante SE, Moreno Mdel P, Hototian SR, Lopes MA, Azevedo $D$, et al. Performance of Brazilian long and short IQCODE on the screening of dementia in elderly people with low education. Int Psychogeriatr 2009 Jun;21(3):531-8.
56. Hancock P, Larner AJ. Diagnostic utility of the Informant Questionnaire on Cognitive Decline in the Elderly (IQCODE) and its combination with the Addenbrooke's Cognitive Examination-Revised (ACE-R) in a memory clinic-based population. Int Psychogeriatr 2009 Jun;21(3):526-30.

57. De Jager CA, Hogervorst E, Combrinck M, Budge MM. Sensitivity and specificity of neuropsychological tests for mild cognitive impairment, vascular cognitive impairment and Alzheimer's disease. Psychol Med 2003;33:1039-50.

58. Howieson DB, Dame A, Camicioli R, Sexton G, Payami H, Kaye JA. Cognitive markers preceding Alzheimer's dementia in the healthy old. J Am Geriatr Soc 1997;45:584-9.

59. Small BJ, Herlitz A, Fratiglioni L, Almkvist O, Backman L. Cognitive predictors of incident Alzheimer's disease:A prospective longitudinal study. Neuropsychology 1997; 11:413-20.

60. Sunderland A, Harris JE, Baddeley AD. Do laboratory tests predict everyday memory? A neuropsychological study. JVerbal Learn andVerbal Behav 1988;22:34157.

61. Baddeley AD, Kopelman MD, Wilson BA. The essential handbook of memory disorders for clinicians. New York: John Wiley \& Sons; 2004.

62. Prestia A, Rossi R, Geroldi C, Galluzzi S, Ettori M, Alaimo G. Validation study of the three-objects-three-places test: a screening test for Alzheimer's disease. Exp Aging Res 2006;32:395-410.

63. Lonie JA, Tierney KM, Ebmeier KP. Screening for mild cognitive impairment: a systematic review. Int J Geriatr Psychiatry 2009 Sep;24(9):902-15.

64. Ravaglia G, Forti P, Maioli F, Servadei L, Martelli M, Brunetti N, et al. Screening for mild cognitive impairment in elderly ambulatory patients with cognitive complaints. Aging Clin Exp Res 2005 Oct;17(5):374-9.

A autora declarou não possuir conflitos de interesse.

\section{ENDEREÇO PARA CORRESPONDÊNCIA}

Manuela Guerreiro

Laboratório de Estudos de Linguagem - Piso 8

Faculdade de Medicina de Lisboa

Hospital de Santa Maria

Av. Prof. Egas Moniz

1649-028 Lisboa

E-mail:mguerreiro@netcabo.pt

\section{ABSTRACT}

\section{COGNITIVE DEFECT AND DEMENTIA SCREENING TESTS: A PRACTICAL PERSPECTIVE}

Early diagnosis of degenerative dementia, particularly Alzheimer's disease (AD), is a matter of great interest to researchers and clinicians. The knowledge of the cognitive profile in preclinical phase of dementias is very important to diagnosis, prognosis, pharmacological and non-pharmacological intervention. General practitioner (GP) in primary care are the most suitable for detecting early cognitive decline, although some studies indicate that dementia is underdiagnosed in primary care. This is due to the limitation of time that GP dispose for the consultations and the lack of habit in the use of cognitive screening tests. The cognitive screening tests should be fast to apply, easy to score and well accepted by patients. In this sense, we will refer some tests for rapid application, citing some of its features and some studies where they were used. The application of a brief test may allow the screening of cases for further detailed neuropsychological examination, because early detection of dementia still in preclinical stages and Mild Cognitive Impairment $(\mathrm{MCl})$ is a public health priority due to high incidence of dementia.

Keywords: Brief Cognitive tests; Screenning; Mild Cognitive Impairment; Mild Dementia. 